\title{
Improving self-management for persons with Parkinsons disease through education focusing on management of daily life: Patients and relatives experience of the Swedish National Parkinson School
}

Carina Hellqvist, Nil Dizdar Segrell, Peter Hagell, Carina Berterö and Märtha SundLevander

The self-archived postprint version of this journal article is available at Linköping University Institutional Repository (DiVA):

http:// urn.kb.se/ resolve?urn=urn:nbn:se:liu:diva-151637

N.B.: When citing this work, cite the original publication.

Hellqvist, C., Dizdar Segrell, N., Hagell, P., Berterö, C., Sund-Levander, M., (2018), Improving selfmanagement for persons with Parkinsons disease through education focusing on management of daily life: Patients and relatives experience of the Swedish National Parkinson School, J ournal of Clinical Nursing, 27(19-20), 3719-3728. https:// doi.org/ 10.1111/jocn.14522

Original publication available at:

https:// doi.org/ 10.1111/jocn.14522

Copyright: Wiley (12 months)

http://eu.wiley.com/WileyCDA/ 


\section{TITLE:}

Improving self-management for persons with Parkinson's disease through education focusing on management of daily life: Patients' and relatives' experience of the Swedish National Parkinson School.

ABSTRACT:

Aims and objective. The two folded aim of this study was firstly to identify and describe experiences valuable for managing daily life after participation in the NPS self-management intervention. The second part was to explore the applicability of the Self- and family management framework by Grey and colleagues for persons with Parkinson's Disease and their relatives. Background. The impact of PD is evident on the lives of both patients and relatives. The National Parkinson School (NPS) is a Swedish self-management program designed for patients and relatives, aiming at teaching strategies helpful for the ability of self-management, in order to promote life satisfaction. Design. Qualitative explorative with inductive and deductive analysis. Methods. Five group discussions with NPS participants were audio-recorded. Verbatim transcriptions were analysed inductively with thematic analysis according to Braun and Clarke, and the findings were then applied deductively to the existing model for patients with chronic disease. Results. Through the first step of inductive analysis three themes capturing the meaning, value and experience of being a participant at the NPS were identified: Exchanging experiences and feeling support, Adjustment and acceptance of PD for managing daily life, and Promoting life satisfaction. The deductive analysis applied the inductive findings to the Self-and family management framework of chronically ill to explore the fit to persons with PD and relatives attending the NPS program. Conclusions. The NPS program is a promising approach for helping persons with PD and their relatives to achieve better self-management of disease and improved life satisfaction. Further evaluations of program outcomes in clinical practice are warranted .Relevance of clinical Practice. Self-management programs like the NPS is a promising approach in facilitating a positive mind-set and outlook on life and gain knowledge to understand, adapt and handle chronic disease, such as PD, better. Keywords: Thematic analysis, Parkinson's disease, self-management intervention. 
What does this paper add to the wider global clinical community?

* This study indicates that self-management interventions with a cognitive approach introducing techniques aimed at handling the psychological impact of disease in the lives of persons with PD and relatives can be helpful in promoting self-care activities and thereby lead to increased lifesatisfaction.

* Attending the self-management intervention as a couple gave knowledge to form a "commonground" enhancing acceptance and mutuality in the spousal relationship.

* General theoretical models guiding research and development of self-management interventions for people with chronic disease can be adjusted and useful for the persons with PD and their relatives.

\section{INTRODUCTION}

Good medical treatment is available for persons affected by Parkinson's disease (PD) but treatment is symptomatic and cannot halt the progression of disease. Many experience fluctuating motor as well as non-motor symptoms of disease that will negatively impact daily life, both for the persons with PD and their relatives. Non-motor symptoms often do not respond as well as motor symptoms to medical treatment (Prakash, Nadkarni, Lye, Young \& Tan 2016), and available pharmacotherapy often results in complications such as behavioural symptoms, motor fluctuations, dyskinesias, and painful dystonia (Kadastik-Eerme et al, 2016). Although persons with PD and their relatives have a long-term need for close and regular contact with healthcare, treatment and care is performed mostly by the person affected with PD and his/her relatives in the community and in everyday life (Beaudet et al, 2015). Accordingly, persons with PD and their relatives want support from health care to develop emotional and cognitive strategies and skills to deal with fear of the future, and to adjust to life with chronic disease in order to live well despite PD. Also guidance and strategies for handling the financial and bureaucratic consequences of the disease and to help with navigating through the health care system are of great importance according to persons with PD (Kleiner-Fisman, Gryfe \& 
Naglie, 2013) Self - management support (SMS) is considered one of the most important activities of outpatient nurses because they have the medical knowledge as well as the close contact with those affected by disease to know how it impacts on their daily life (Chenoweth, Gallagher. Sheriff, Domoghue \& Stein-Parbury 2008; Elissen et al, 2013)

Self-monitoring is considered an important skill to manage daily life. Self-monitoring includes observations, assessment and monitoring of physical symptoms and activities of daily living, and cognitive processes leading to self-awareness. This information can form the basis for self-care activities and for consultation with health care professionals (Wilde \& Garvin 2007). Self-monitoring is one component in the broader concept of self-management defined as the ability of the person, in union with family, community and healthcare professionals to manage symptoms, treatments, lifestyle changes and psychosocial, cultural and spiritual consequences of health conditions (Wilkinson \& Whitehead, 2009). The concept of self- management is frequently used in the literature concerning people living with chronic diseases (Richard \& Shea 2011). Self-management is considered a complex and multidimensional phenomenon, linked intimately in almost all patients to family management as it occurs in the context of family (Grey, Knafl \& McCorkle, 2006; Grey, Schulman-Green, Knafl \& Reynolds, 2015).There are facilitators and barriers to self-management as well as the processes leading to self-management behaviours. A person's ability in self-management will have outcomes both for the person as well as for the relatives and health care. Self-management interventions are designed to help persons with chronic diseases deal with different aspects of the disease, such as symptom control, medication and emotional reactions of disease in daily life.

\section{BACKGROUND}

In 2002, the EduPark project was initiated in seven European countries. The intention of EduPark was to develop and implement an educational program for persons with PD and their relatives. EduPark resulted in an educational program called "Patient education for persons with PD and their Carers" (PEPP) (Smith Pasqualini \& Simons, 2006) Inspired by PEPP, a Swedish patient educational program 
called The National Parkinson School (NPS) was developed in 2013. Both the NPS and PEPP are based on knowledge and experiences from cognitive behavioural therapy and were developed through collaboration among healthcare providers, researchers, the pharmaceutical industry and patient organizations. Similar to PEPP, the aim of the NPS is to provide persons with PD and their relatives with tools and strategies to increase their ability to manage daily life with disease and to promote life satisfaction (Smith et al, 2006). Self-monitoring as well as self-management are central concepts of the PEPP and the NPS. The NPS consists of seven sessions where topics concerning PD and life with chronic disease are in focus through educational sessions, group discussions and home assignments e.g. managing stress in daily life (see Appendix 1).

Since the development of the NPS in 2013 it has been implemented in clinical practice in several neurologic and geriatric clinics in Sweden. However, it is unknown how the NPS program is perceived by participants in terms of the educational content of the program or regarding the experience of being a participant in the NPS. The two folded aim of this study was firstly to identify and describe experiences valuable for managing daily life after participation in the NPS selfmanagement intervention. The second part was to explore the applicability of the Self- and family management framework by Grey and colleagues for persons with Parkinson's Disease and their relatives.

\section{METHODS}

The study has a qualitative explorative design with two step analyses using both inductive and deductive approach. Ethical approval was obtained from the Swedish ethical advisory board (2014/497-31). Written consent for permission to collect data was obtained from the five clinic directors in charge. Study participants were given both written and oral information and informed consent was obtained from both participants with PD and their relatives. The study was carried out in accordance with the Declaration of Helsinki (World Medical Association, 2013). 
3:2 Data Collection. Participants were recruited from five separate outpatient geriatric and neurologic clinics in the middle and southern parts of Sweden. All participants were selected by staff on a solely clinical basis for participation in the NPS. The data collection took place between August 2015 and June 2016. Demographic information and disease history were obtained by a questionnaire administered to persons with PD and relatives at the onset of the NPS. The last session of the NPS (session 7: " My life with Parkinson's Disease" ) was audio-recorded in its whole and transcribed verbatim. The session is standard and a part of the clinical NPS program. During this session, the participants are asked to look back at the contents and techniques discussed in the previous NPS sessions, under the guidance and presence of the NPS educator. It includes a reflection by the participants on the educational program as a whole and the expected use of the knowledge and technique attained through NPS in their daily lives ahead. The session was not in any way modified for the purpose of this research study as the aim was to capture the clinical reality and value of the NPS program. Before the start of the session the researcher would give a short information about the purpose of and use of this recording for research and place a recording device in the room. The researcher would then leave the room so the dynamics and interactions of the group would not be affected by being present. All five group discussions were recorded in a conference room setting at the participating clinic used for meetings and/or patient-educational purposes. Each group discussion lasted for about two hours and each generated between 25-40 pages of transcribed text

3.3 Data Analysis. The data were analysed in two steps; first an inductive analysis, using thematic analysis, which is a flexible method of identifying and analysing patterns within a dataset (Braun \& Clarke, 2006) was performed. Each group interview was analysed by the three researchers independently $(\mathrm{CH}, \mathrm{CB}, \mathrm{MSL})$, identifying initial codes that were grouped into tentative themes. The 
themes and included codes were further discussed within the research team, resulting in a thematic map with six themes (Figure 1).

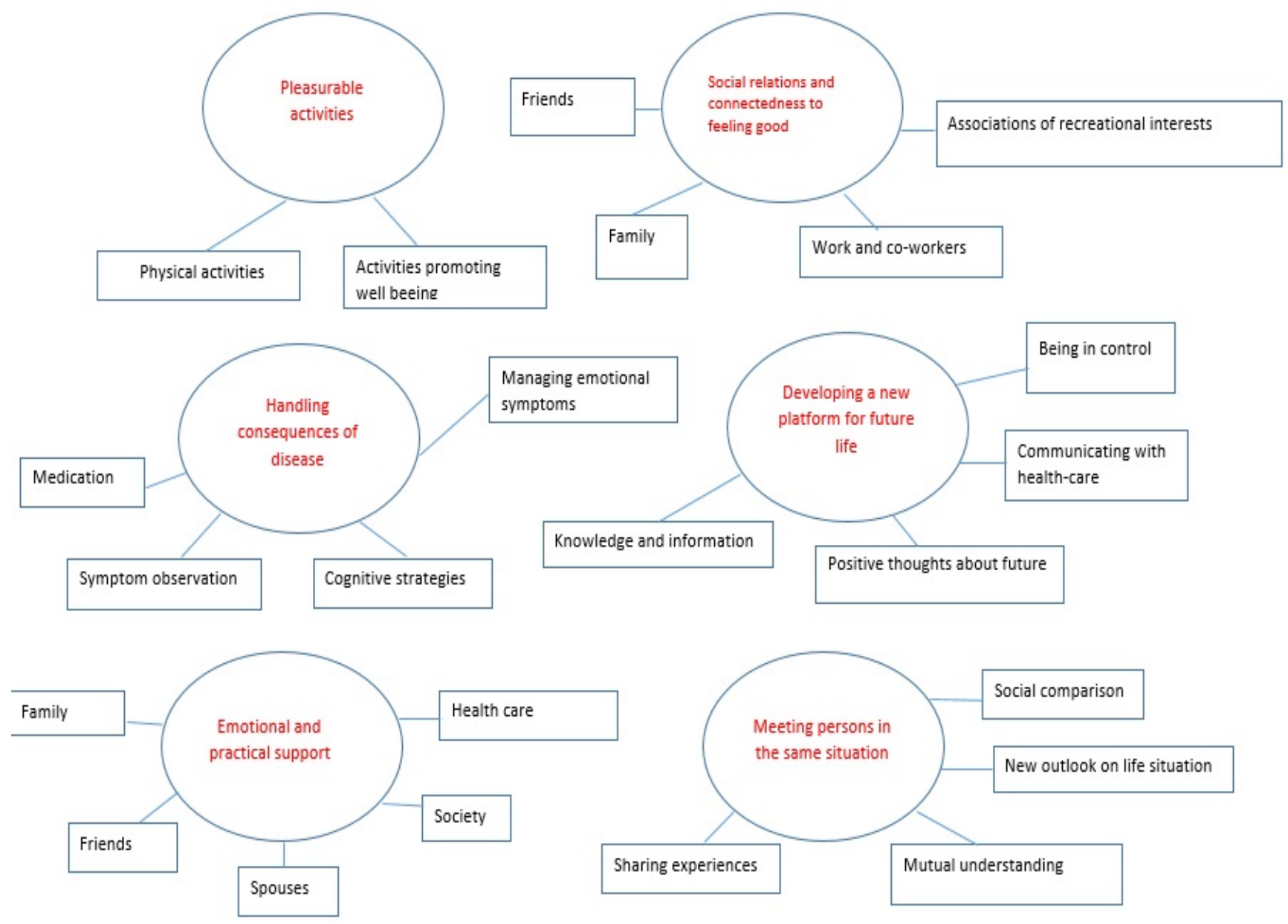

Figure 1: Example of the analysis process developing a thematic map with six identified themes and codes.

Through the ongoing analysis, the contents of each identified theme were further clarified. Through this process, three final themes of interest to the research question were identified. The meanings of these themes were verified by actively re-reading the five transcribed group discussions several times to get a feeling for the entire data set. The 15 criteria for good thematic analysis described by Braun and Clarke (Braun \& Clarke, 2006) were adopted. 
The findings from this first step inductive analysis was then applied in a deductive manner (Braun \& Clarke, 2006) to an already existing framework used to guide self-management support research and interventions for patients with chronic disease, the Self and family management framework by Grey and colleagues $(2006,2015)$. This framework is a model of self-management for persons with chronic illness and their families, identifying facilitators and barriers of self-management as well as processes of self-management and proximal and distal outcomes of self-management for the individual, family and society.This deductive application was done because researchers wanted to test the applicability and feasibility of the framework for the specific population of persons with PD as it has not been done before. If applicable, the framework of Gray and colleagues could be used to guide research and to design and improve self-management interventions such as the NPS for persons with PD and relatives in the future.

Both steps of analysis, first the inductive thematic analysis and then the deductive application to the framework, were first performed by the three researchers independently. The findings were then discussed, compared and checked back with the transcribed texts, and then finalized all researcher together. This form of researcher triangulation strengthens the validity and rigour of the findings of this study (Patton, 2002).

\section{RESULTS}

In total, 42 participants were included in the study, 25 persons with PD; (14 men and 11 women) and 17 relatives; (9 men and 8 women). The educational levels of the participants were primary school ( $n=13)$, high school diploma $(n=12)$ and college/university degree $(n=17)$. All participants except one were married. PD duration varied between, less than one year up to 13 years. The persons with PD were mainly in mild to moderate Hoehn and Yahr (1967) stages of disease (Stage $1 n=3$, Stage $2 n=13$ stage $3 n=9$ ). No one was in stage 4 , severe disease. 
Through the inductive analysis three major themes capturing the meaning, value and experience of being an NPS participant were identified and described in a thematic map (Figure 2)

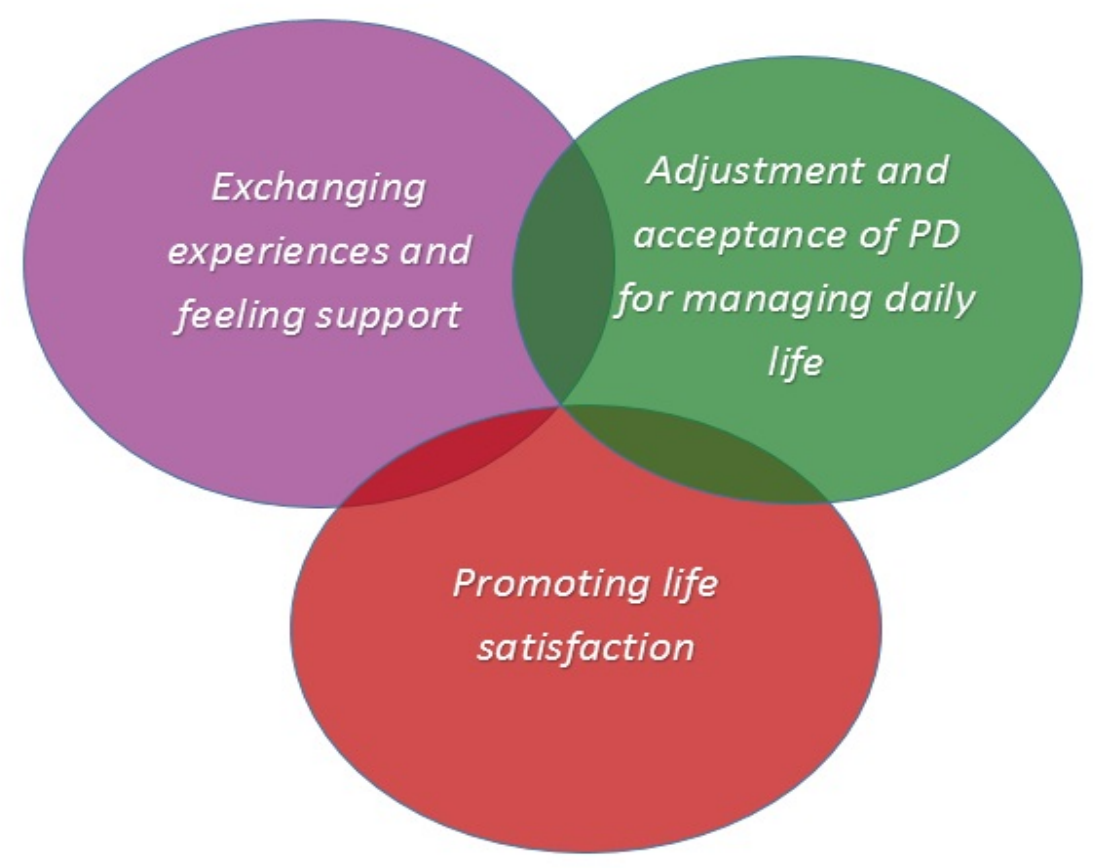

Figure 2: Final thematic map with the three major themes illustrating the meaning, value and experience of being an NPS participant.

\section{4:1 Exchanging experiences and feeling support}

Participating in a group with others in the same situation dealing with the physical and emotional challenges of PD, either as affected by disease or as relatives, was in itself considered very valuable and positive. It was comforting and reassuring to realize that "I'm not the only one in this situation" and this made participants feel less isolated. Although participants also came to realize that the symptoms of PD could vary considerably between individuals there was also a common ground resulting in a fruitful exchange of knowledge and experiences of handling daily life and managing symptoms. Sharing practical advice about medication, exercise and sleep was also common and much appreciated. Meeting in the same group for several weeks made participants feel connected to 
each other, and getting to know each other also made participants feel comfortable in sharing experiences and opinions. In the group, participants made statements to give each other emotional support and promote the feeling that there was a hopeful and good future to come in spite of the presence of PD in their lives. Several participants expressed the benefits of available medical treatment for PD and stated that although chronic, the disease was not fatal. The sharing of emotional support was considered very helpful and important among the persons with PD, as well as between their relatives.

"My feeling is that this group has been a helping hand because it has been very heavy trying to carry it all by myself...It's been a life-saver... Thanks to this NPS education and the exercise sessions I feel that me and my husband have a future to look forward to. I believed it looked very dark before... and it was so hard trying to carry it all by myself" (Group 1 row 1110-1115)

Many participants expressed the benefit of attending the NPS together as a couple. To obtain the same skills and knowledge would give them a mutual understanding and common ground and would also open up discussions that they could continue and develop later at home. However, some of the relatives expressed a wish and need for having a "relatives-only" session in the NPS. They felt there might be issues they wanted to discuss that might be too sensitive to talk about with the relative present or in a larger group. Several relatives expressed a desire to get more knowledge on how to help and to be a support and resource for their partners in managing disease.

Social interaction with other people in society was recognized as being both supportive but also at times discouraging for the persons and relatives dealing with PD as a part of daily life. Family and good friends could be a huge resource, offering emotional support, social interaction and help with more practical chores. Spending time with friends and family was considered a relief and made it possible to forget about the disease and its symptoms for a while.

"I've been thinking... It might not be the activity in itself but the social interaction 
surrounding the activity. As I told you, we are a couple of friends meeting to

prepare good food together. But the cooking itself has actually less importance,

it's just getting together... that is what is important!" (Group 3 row 524-527)

Willingness to talk about PD when asked was a strategy considered helpful when interacting with society. Articulating the presence of the disease would often bring about more tolerance and help if needed, for example on the bus or in the grocery store. The timing and amount of information given about the condition was dependent upon who was asking and in what situation the person was. The presence of PD could in some cases make people around feel uncertain and feel pity towards the person. It was sometimes associated with the social stigma of "being ill". Interactions with healthcare staff were initiated to gain knowledge and advice about PD and medications and also information on available financial support and aids. Long-term good relations and easy access to healthcare staff also provided the opportunity for emotional support and confidence both for the persons with PD and their relatives.

\section{4:2 Adjustment and acceptance of PD for managing daily life}

The technique of self-monitoring included in the NPS, i.e. to observe and eventually make changes in behaviour or as a way of dealing with the physical and emotional symptoms of PD was new and somewhat hard to grasp for some of the participants. If the concept was not explained more thoroughly by the educators i.e. in terms of "keeping a diary", the meaning of self-monitoring was unclear to some. Other participants seemed to get the idea of the concept but instead reflected on the difficulties of changing one's behaviour even if you were aware and had observed yourself. Inner motivation and participation in a group to increase physical activity were seen as promoters of change. Participants in the groups that already had several years of experience living with PD commented that the concept of self-monitoring and techniques to handle stress, anxiety and other 
symptoms were not new to them. Over the years, they had already figured out strategies similar to those covered in the NPS, e.g. keeping a diary to observe themselves. The practice of keeping a diary had helped them to learn to recognize symptoms of disease as well as their own feelings and actions.

"The first 5-6 years of my disease I wrote a diary. I wrote down what I was

thinking and feeling and what I could and could no longer do, and coming to

conclusions, and I could change the way I was feeling. It was very helpful

for me and gave me perspective on my situation.... I could make action plans. // That

kept me in contact with how I should live my life instead of just accepting that

everything was miserable because I realized I was in charge of how it was going

to be. It was really good for me" (Group 1 row 569-571// row 578-580)

Experienced patients and relatives appreciated the NPS sessions as a way of meeting others in the same situation. However, the contents of the educational sessions were considered to have been of more use to them in the first years after the diagnosis of PD. Other statements of novice patients and relatives seemed to confirm that they perceived the strategies of self-monitoring and techniques to handle stress and anxiety introduced during the NPS as helpful for them.

"... and this education as a whole has helped me to take on my illness in a more powerful way. Maybe it's because I'm relatively newly diagnosed... for me... this education gave me hands-on tangible things to do for myself. That felt good for me" (Group 3 row 41-43)

The relaxation exercise at the end of each session provided time to reflect upon what had been discussed and learned earlier in the session and made participants feel that this could settle inside the body and mind, and that they could leave the session with a feeling of inner peace. 
Accepting disease was seen as a gradual process of going through all kinds of emotions from denial, sadness and anger to slowly accepting and eventually looking with new hope and positive thoughts at the future. Trying to have a positive mind-set and outlook on life and the present situation was recognized as a very important strategy for accepting and living life as well as possible. Finding strategies to handle uncertainty about the disease progress and fear of the future was necessary. Thinking through situations that might cause stress and anxiety, and trying to make a plan to change the negative thoughts that usually arose in that situation to more positive and calming ones were considered important, appreciated and useful strategies applicable in the present life situation for most participants. Seeking information was one strategy for adapting to life with PD. Having knowledge was like a new way to understand and manage the changed conditions in life now and in the future. The sources of information were many and participants actively sought information from books and different internet sites and discussion forums. Information provided by medical staff such as the PD nurse specialist and physician was considered very valuable. A barrier to accessing knowledge from the health care staff was lack of time during visits and long intervals between visits. Health care staff were valuable for discussing and consulting about symptoms, and for help in choosing and adjusting medical treatment.

\section{4:3 Promoting life satisfaction}

Being active in life was considered crucial for life satisfaction and was extensively discussed in all the groups. Activities could be of different types, including physical or mental activities as well as spiritual and cultural ones. Discovering and enjoying the little things in everyday life was something that some of the participants were doing in their daily lives while others recognized it as an area for improvement. Actively focusing on the small things that generated feelings of happiness in daily life also helped, and hindered feelings of sadness and isolation that could lead to depression. 
"When I'm not feeling well and I feel sad... then I think to myself... God damn...

there are a lot of things that are good! And when someone asks me how I'm feeling I say "Well there are a lot of good things going on in my life!". And saying that makes me think things through... What are the good things in my life? I think of specific episodes that have been good and that are good and it's not very hard to find things. There are so many good things happening every day! They might be the smallest things but they are damned good for me! Like my wife giving me a goodmorning hug. That's absolutely wonderful!" (Group 2 row 373-380).

Activities that promoted feelings of happiness and relaxation often involved spending time with other people. Meeting family and particularly grandchildren was much appreciated. Other enjoyable experiences involved outdoor activities such as gardening and walking in the woods as well as creative activities such as painting, cooking or baking. Also reading books, listening to music and singing were activities enjoyed by many.

The importance of physical activity for persons with PD is emphasized very strongly by healthcare as it improves long-term prognosis and helps maintain and improve bodily functions, mobility and muscular strength. Most participants in the NPS already had contact with a physiotherapist and were engaged in training at least once a week. Mental barriers to physical activity were identified by participants as lack of energy and motivation. Some also had physical barriers hindering them, such as pain or poor balance. Physical activities performed in a group were motivating and those living with a spouse could motivate each other to initiate and perform physical activities. Relatives recognized this as an area where they could be active in motivating and helping the spouse with PD to feel and function better. Also, cognitive strategies such as written schedules and weekly plans 
could serve as reminders and motivators. The effect of physical activity was obvious to many of the participants.

"Everybody is always stressing the importance of physical activity and training. And I've been like... okay... Does it really have an impact at all? Well now we've got proof that it works! I was out walking with her (wife with PD) today and she has never walked so fast! I had a hard time keeping up!" (Group 1 row 934-938)

The physical activities performed for pleasure and leisure varied and could include walking, biking, swimming, going to the gym or attending group exercise classes.

4: 4 The inductive findings applied to the Self-and family management framework of chronically ill to explore the fit to persons with PD and relatives attending the NPS program.

Through the deductive analysis the three themes inductively identified and of interest to the research question were now applied and sorted into the Self- and family management framework. To use the Self-and family management framework by Grey and colleagues was a way of better understanding the components and outcomes of the NPS program explored in this study. The result is a model visualizing the theory's applicability to the persons with PD and relatives participating in the NPS (Figure 3). 


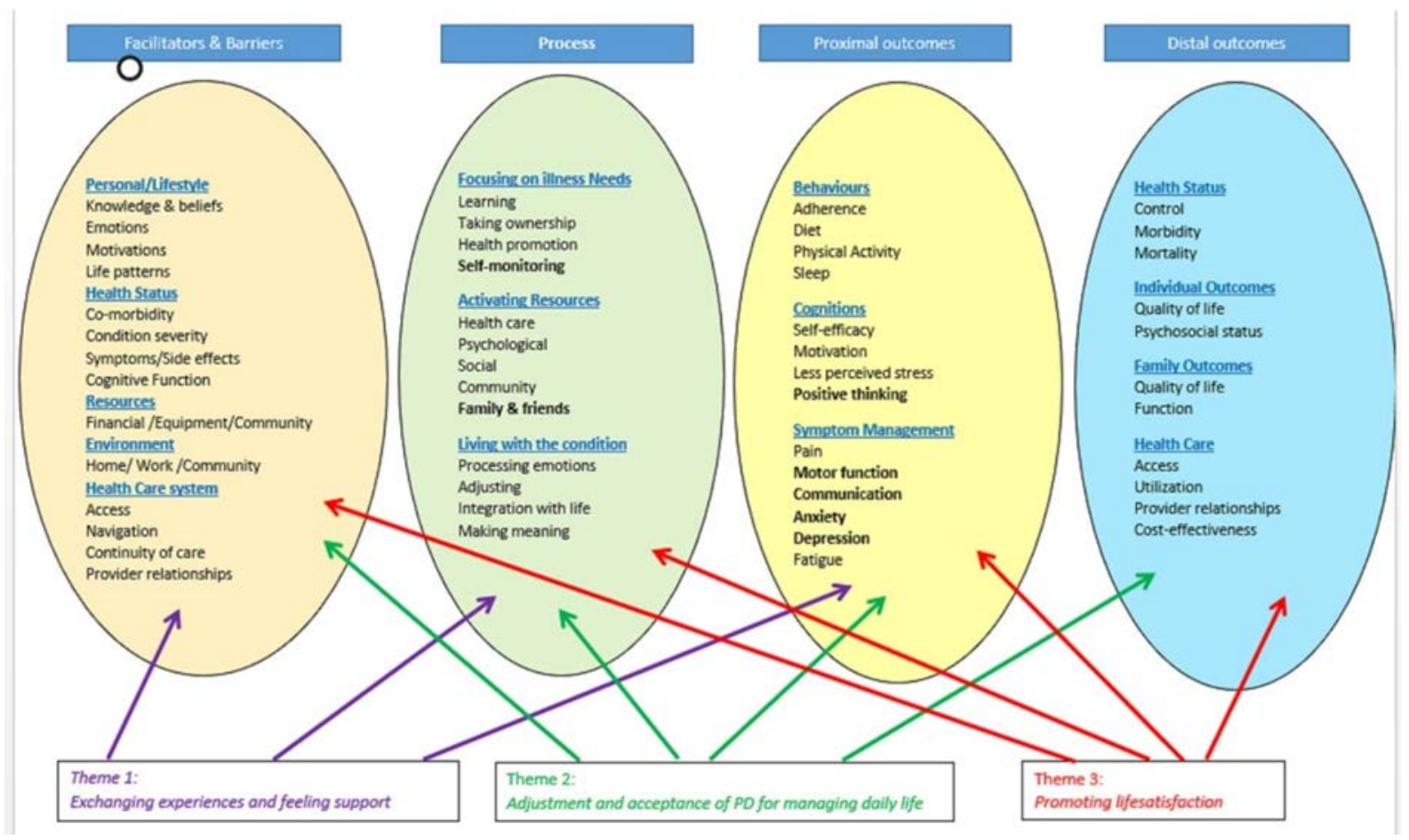

Figure 3: Exploring Self- and Family management framework (Grey $M$ et al, 2015) applicability to the persons with PD and relatives participating in the NPS

The results show that the general framework for self-management in people with chronic disease presented by Grey et al. (2015) is also applicable to persons affected with PD and their families. Some factors needed to be clarified, modified or added regarding PD. For example self-monitoring (focusing on illness needs) is a strategy taught in the NPS as a skill to better understand and interpret symptoms of disease leading to a better basis for self-management behaviours and improved communication with health care providers. Family and friends were added to the model as an activating resource since it was evident that they were much involved in motivating and promoting health-related behaviours positive for self-management. Positive thinking (Cognitions) was also added as a strategy that was much used and well accepted by participants of the NPS. Common PDspecific symptoms connected to the nature of this neurodegenerative disease; motor function, communication, anxiety and depression were added. Factors not presented in our visualisation of the original model, were also not present in our data from the PD population attending the NPS, such as 
'Spiritual ' as an activating resource since this was not mentioned at all as a factor for patients or relatives in this study. Also, the proximal outcome biomarkers were not presented since no measurable biomarkers are monitored or likely to change depending upon a person's ability to selfmanage in persons with PD

The three themes identified through the first inductive step could be identified and applied to the theory. In the content of each theme factors were present that were relevant in all the areas as facilitator, barriers, processes or outcomes. For the theme of Exchanging experiences and feeling support the distal outcome variables were not identified or obviously expressed in the data set.

\section{DISCUSSION}

The results of this study show that the NPS program is a promising approach helping persons with PD and their relatives to achieve better self-management of disease and better life satisfaction. Selfmanagement programs are viewed as complex interventions affecting many areas of a person's life. This complexity mirrors the difficulties in identifying one single aspect that makes self-management valuable for the participants.

The NPS is a self-management intervention designed to suit persons with PD and their family members in a Swedish cultural and health care context. The NPS covers some aspects of PD and its treatment but mainly targets cognitive and behavioural strategies of self-management in daily life. These strategies were evident throughout the data and the three identified themes. Positive thinking and changing negative and stressful thoughts to more positive ones are discussed in several sessions of the NPS and the participants seemed to adapt this idea and find this approach to future life beneficial. A positive outlook and optimism about the future have been shown to improve quality of life, help one fell in charge of one's own health and future, and improve self-reported disease disability (Gison, 2015. Gruber-Baldini, 2009. Johnston, 2012). Being in a group and meeting people in the same situation, sharing experiences and receiving and giving support were seen as among the most valuable things about the NPS. The feeling of not being alone in the struggle of living with and 
handling daily life with PD reduced feelings of isolation. Meeting in a group of peers gave a sense of fitting into a new context that strengthened and clarified a new identity and sense of self. For those who had lived with PD for many years the social interaction in a group of peers was considered the most valuable aspect of the NPS, even though the strategies and knowledge taught were not new to them. The value of peer support and group interactions in self-management programs has also been recognized for other chronic disorders (Johnston, Irving, Mill, Rowan \& Liddy, 2012; Jormfelt, Rask, Brunt, Bengtsson \& Svedberg, 2012)

Another issue that was prominent and clearly articulated by the participants in all five groups, although not touched upon or mentioned in the NPS in the educational material or by educators, was the strategy of downward social comparison in order to feel more positive about one's own situation. Statements such as "I have had worse information to handle before", "There is always someone worse off than me" and "We will not die from this disease" were present in all groups. Also social comparison, but the other way around, was evident for some of the novice participants as they found other members of the NPS groups to be good examples and an inspiration on how to handle disease and proof that a good life is possible despite PD. Downward and upward social comparisons have also been found in self-management groups for persons with other chronic diseases (Rogers, Gately, Kennedy \& Sanders 2009). The use of self-monitoring to handle PD symptoms and to promote self-management was new to some while others had been using it for several years. The participants found self-monitoring helpful to "sort symptoms out" and to make symptoms more understandable and easier to handle. Self-monitoring could be seen as creating a new platform for understanding one's situation and for strengthening feelings of being in control, thereby making adaption to life with PD easier. It also showed participants in the NPS their own responsibility to actively engage in self-management to promote well-being in life. One expected outcome of the NPS is an improved ability to handle PD in daily life, as well as improved life satisfaction and perceived health. Previous studies have shown mixed outcomes with improvements in handling daily life and improve health and no changes at all in health-related outcomes following participation in the PEPP 
program (A'Campo, Spliethoff-Kamminga \& Rood, 2011; A'Campo,Wekking, Spliethoff-Kamminga, Le Cessie \& Roos, 2010). This may also have been true for the participants in this study but this was not assessed. Life satisfaction for persons with PD and their relatives participating in the NPS mainly appeared to be connected to performing enjoyable activities that generated feelings of happiness and distracted them from the disease for a while. Physical activity had the benefit both of being a mediator of disease symptoms leading to improvement of the medical condition, and was also often considered as enjoyable and promoting life satisfaction. An increase in physical activities as well as other enjoyable activities has also been observed in other chronic conditions following participation in self-management programs (Johnston et al, 2012).

The most prominent difference between the NPS and the PEPP is that patients and relatives are in a group together during all seven sessions of the NPS whereas this is not the case with PEPP. The reason for being in a group is to strengthen feelings of mutuality as a couple "being in this and handling it together". Several studies show that strong mutuality in the spousal relationship is associated with better mental health of both partners, reduced caregiver burden and improved relative quality of life (Beaudet \& Duchame, 2013; Beudet et, 2015; Tanji et al, 2008; Wressle, Engstrand \& Granérus 2007). In this study the couple approach was generally appreciated but some relatives expressed a need for a "relatives-only" session to discuss more sensitive topics. There may also be a need to investigate how the NPS could be made more useful and of interest to relatives and persons with PD with a longer disease duration. There is published evidence that life satisfaction for persons with PD may be promoted through interventions like the NPS that facilitate understanding and handling of the disease. Interventions aiming at the more psychosocial and cognitive approach of living with PD are more influential on satisfaction with life than the severity of disease in itself ( Rosengren, Brogård, Jacobsson \& Lexell, 2016; Robottom et al, 2012). While this study is the first evaluation of the NPS, further studies are needed to assess its outcomes from the participant's perspective, as well as the cost effectiveness and the economic rationale for performing this type of intervention. 
The methodology used in this study - an inductive approach in analysing the transcribed texts and identifying themes, followed by a deductive approach comparing and applying the identified themes to an existing framework - has given this study two dimensions. First, it captured the views and opinions of NPS participants themselves in three distinct themes identified from the transcriptions of group discussions with no pre-decided categories in mind. Then, it looked at the three identified themes again and applied the existing Self - and family management framework (Grey et al, 2006; Grey et al, 2015), which revealed that this framework of self-management for other chronic diseases appears to be applicable also for persons with PD and their relatives. Most of the factors, progresses and outcomes described in the original model could also be seen in the data of persons with PD and relatives involved in the NPS; however, some of the factors in the original framework were not relevant to this group. Nevertheless, the framework seems to be a robust one and can be used as a guide for health care staff as well as educators and researchers planning self-management interventions or studies for persons with PD and their relatives.

The strength of this study is that it reflects the participants' own views and opinions about the NPS program. This gives a good sense of what is valuable to them and what should be kept in the program but also what were seen as weaknesses and should be considered areas for improvement in the NPS program. There is evidence that interventions targeting cognitive processes, inner motivation and self-efficacy are more successful in improving self-management skills than solely educational approaches (Van Hooft, Been-Fahmen, Ista, Van Staa \& Boeijeh, 2016). The NPS is an intervention of the first kind and therefore it is likely to have a positive outcome on participants' ability to handle daily life with PD. Although the participants clearly appreciated the NPS, this study cannot answer to what extent the NPS is successful in increasing participant's ability to manage life with PD and promote life satisfaction. Further longitudinal studies using other research methods 
should address these and other outcomes. One approach to investigate the effectiveness and outcomes of a self-management program instead of looking at changes in participant-reported parameters is to consider general measures of the intended outcomes of SMS interventions. Specific outcome measures, e.g. pain or fatigue, can remain unchanged and are not related to an increased ability to self-manage (Osborne, Batterham \& Livinston 2011). Another weakness of this study is that the results do not represent the most severe stages of PD. Such patients often have major cognitive and physical limitations and many of them live in nursing homes (Porter, Henry, Gray \& Walker,2009)

\section{CONCLUSION}

This study has shown that the Swedish NPS self -management program for persons with PD and their relatives is appreciated by participants. From the participants' view the benefits of attending the NPS were social connectedness and support from people in the same situation, as well as improved social connection to family, society and health care. They also gained strategies and cognitive techniques to monitor symptoms and change behaviours, adapting a positive mind-set and outlook on life and gaining knowledge in order to understand, adapt to and handle the disease better. The NPS program also gave an increased awareness of the need to prioritize activities that promote feelings of well being and satisfaction with life. Further evaluations of the outcomes of the NPS in clinical practice are warranted.

\section{RELEVANCE TO CLINICAL PRACTICE}

This study adds on to the scientific evidence of the positive outcomes of self-management interventions for persons with PD and relatives in clinical care. Self- management interventions like the NPS can be very helpful for persons with PD as well as for the relatives in order to learn techniques to monitor symptoms of disease, leading to an improved ability to perform the self-care activities needed to manage both physical and psychological symptoms of PD. Nurses need to 
approach the persons with PD and their spouses with the attitude that their narrative of the experiences of dealing with the consequences of disease in daily life is important. Nurses should encourage the formation of a therapeutic partnership with the persons with PD and relatives and communicate that they are vital partners in a team; striving for better health and well-being. The persons with PD and their spouses are able and willing to participate in their own care and treatment if they are allowed to do so. As an educational self-management intervention the NPS is strengthening the participants' self-care capability and nurses have a mission to preserve this ability by supporting and inviting persons with PD and their spousal to be active and involved in shared decision making in their own care. A spousal approach in the care delivered can promote mutual understanding and knowledge and strengthen mutuality of the relationship. Meeting others in the same situation during the NPS education was encouraging and introduced a new social-network of support for persons with PD and relatives. The many benefits of self-management interventions for persons with PD and relatives emphasizes that it should be integrated and offered as a vital part of good clinical care. 


\section{REFERENCES}

A'Campo, LE., Spliethoff-Kamminga ,NG., \& Roos, RA. (2011). An evaluation of the patient education programme for Parkinson's disease in clinical practice. Int J Clin Pract., 65, 1173-9. DOI:

10.1111/j.1742-1241.2011.02765.x

A'Campo, LE., Wekking, E.M., Spliethoff-Kamminga, NGA., Le Cessie, S., \& Roos, RAC. (2010).The benefits of a standardized patient education program for patients with Parkinson's disease and their caregivers. Parkinsonism \& Related Disorders, 16, 89-95.

https://doi.org/10.1016/j.parkreldis.2009.07.009

Beaudet, L., Duchame, F., LÈcyer, N., Chouinard, S., Jodoin, N., \& Panisset, M. (2015). Development and evaluation of a dyadic intervention for elderly couples living with moderate-stage Parkinson disease. Applied Nursing Research,28 , 21-27. DOI: 10.1016/j.apnr.2015.02.004

Beaudet, L., \& Duchame, F. (2013). Living with Moderate-Stage Parkinson Disease: Intervention Needs and Preferences of Elderly Couples. Journal of Neuroscience Nursing, 45, 88-95. DOI: 10.1097/JNN.0b013e3182828ff4

Braun, V., \& Clarke, V. (2006). Using thematic analysis in psychology. Qualitative Research in psychology, 3, 77-101. DOI:10.1191/1478088706qp063oa

Chenoweth, L., Gallagher, R., Sheriff, J. N., Donoghue, J. \& Stein-Parbury, J. (2008). Factors supporting self-management in Parkinson's disease: implications for nursing practice. International Journal of Older People Nursing, 3, 187-193. DOI: 10.1111/j.1748-3743.2008.00123.x

Elissen A., Nolte E., Knai C., Brunn, M., Chevreul, K,, Conklin, A....\& Vrijhoef, H. (2013). Is Europe putting theory into practice? A qualitative study of the level of self-management support in chronic care management approaches. BioMed Central Health Services Research, 13, 117. DOI:

$10.1186 / 1472-6963-13-117$ 
Gison, A., Rizza, F., Bonassi, S., Donati, V., \& Giaquinto, S. (2015). Effects of dispositional optimism on quality of life, emotional distress and disability in Parkinson's disease outpatients under rehabilitation. Functional Neurology, 30, 105-111. DOI: 10.11138/FNeur/2015.30.2.105

Grey, M., Schulman-Green, D., Knafl, K., \& Reynolds, N.( 2015). A revised Self-and Family Management Framework. Nursing Outlook, 63, 162-170. DOI: 10.1016/j.outlook.2014.10.003.

Grey, M., Knafl, K., \& McCorkle, R.(2006). A framework for the study of self-and family management of chronic conditions. Nursing Outlook, 54, 278-286. DOI: 10.1016/j.outlook.2006.06.004 Gruber-Baldini, AL., Ye, J., Anderson, K.E., \& Schulman, LM.( 2009). Effects of optimism/pessimism and locus of control on disability and quality of life in Parkinson's disease. Parkinsonism and Related Disorders, 15, 665-669. DOI: 10.1016/j.parkreldis.2009.03.005

Hoehn, MM \& Yahr, MD. (1967). Parkinsonism: onset, progression and mortality. Neurology 17, 42742.

Johnston, S., Irving, H., Mill, K., Rowan, MS., \& Liddy, C. (2012). The patient's voice: An exploratory study of the impact of a group self-management support program. Family Practice, 13, 65. DOI: $10.1186 / 1471-2296-13-65$

Jormfelt, H., Rask, M., Brunt, A., Bengtsson, A., \& Svedberg, P. (2012). Experiences of personcentered health education group intervention- a qualitative study among people with a persistent mental illness. Issues Ment. Health Nurs., 33, 209-216. DOI: 10.3109/01612840.2013.775614 Kadastik-Eerme, L., Muldmaa, M., Lilles, S., Rosenthal, M., Taba, N., \& Taba, P. (2016). Nonmotor Features in Parkinson's Disease: What Are the Most Important Associated Factors? Parkinson's Disease, Article ID 4370674, 8 pages, 2016. DOI:10.1155/2016/4370674 
Kleiner-Fisman, G., Gryfe, P., \& Naglie, G.(2013). A Patient-Based Needs Assessment for Living Well with Parkinson Disease: Implementation via Nominal Group Technique. Parkinson's Disease, 2013, Article ID 974964, 6 pages. DOI: 10.1155/2013/974964

Osborne, RH., Batterham, R., \& Livingston, J. (2011). Chronic Disease Self-Management Support across Settings: The International Experience of the Health Education Impact Questionnaire Quality Monitoring System. Nurs Clin North America, 46, 255-270. DOI: 10.1016/j.cnur.2011.05.010

Patton, M Q. (2002). Qualitative Research \& Evaluation methods. Sage Publications, Thousand Oaks.

Porter, B., Henry, S R., Gray, W K., \& Walker, R W. (2009). Care requirements of a prevalent population of people with idiopathic Parkinson's disease. Age Ageing, 39, 57-61. DOI: 10.1093/ageing/afp199

Prakash, K M., Nadkarni, N V., Lye, W K., Yong, M H., \& Tan, E K. (2016). The impact of non-motor symptoms on the quality of life of Parkinson's disease patients: a longitudinal study. European Journal of Neurology, 23, 854-860. DOI: 10.1111/ene.12950

Richard, AA., \& Shea, K. (2011). Delineation of self-care and associated concepts. J Nurs Scholarsh.,43, 255-64. DOI: 10.1111/j.1547-5069.2011.01404.x

Robottom, BJ., Gruber-Baldini, AL., Anderson, KE., Reich, SG., Fishman, PS., Weiner, WJ., \& Schulman, LM. (2012). What determines resilience in patients with Parkinson's disease? Parkinsonism and related disorders. 18: 174-177. DOI: 10.1016/j.parkreldis.2011.09.021

Rogers, A., Gately, C., Kennedy, A., \& Sanders, C. (2009). Are some more equal than others? Social comparison in self-management skills training for long-term conditions. Chronic Illness, 5, 305-317. DOI: $10.1177 / 1742395309350384$ 
Rosengren, L., Brogård, C., Jacobsson, L., \& Lexell, J. (2016). Life satisfaction and associated factors in persons with mild to moderate Parkinson's disease. Neurorehabilitation,39, 285-294. DOI:

\subsection{3/NRE-161359}

Smith Pasqualini, M., \& Simons, G. (2006). Patient Education for People with Parkinson's Disease and their carers. John Wiley \& sons, Chichester.

Tanji, H., Anderson, K. E., Gruber-Baldini, A. L., Fishman, P. S., Reich, S. G., Weiner, W. J., \& Shulman, L. M. (2008) Mutuality of the marital relationship in Parkinson's disease. Mov. Disord., 23, 18431849. DOI: $10.1002 / \mathrm{mds} .22089$

Van Hooft, S .M., Been-Dahmen, J.M. J., Ista, E., Van Staa, A., \& Boeijeh, R. (2016). A realist review: what do nurse-led self-management interventions achieve for outpatients with a chronic condition? Journal of Advanced Nursing, 73, 1247-1532 DOI: 10.1111/jan.13189.

Wilde, MH., \& Garvin S. ( 2007). A concept analysis of self-monitoring. Journal of Advanced Nursing, 57, 339-350. DOI: 10.1111/j.1365-2648.2006.04089.x

Wilkinson, A., \& Whitehead, L. (2009). Evolution of the concept of self-care and implications for nurses: A literature review. International Journal of Nursing Studies, 46, 1143-1147.

World Medical Association. (2013). WMA Declaration of Helsinki- Ethical Principles for Medical Research Involving Human subjects. http://www.wma.net/en/30publications/10policies/b3/ Wressle, E., Engstrand, C., \& Granérus, AK. (2007). Living with Parkinson's disease: Elderly patients' and relatives' perspective on daily living. Australian Occupational Therapy Journal, 54, 131-139. DOI: $10.1111 / \mathrm{j} .1440-1630.2006 .00610 . x$

\section{FIGURE LEGENDS}

Figure 1: 


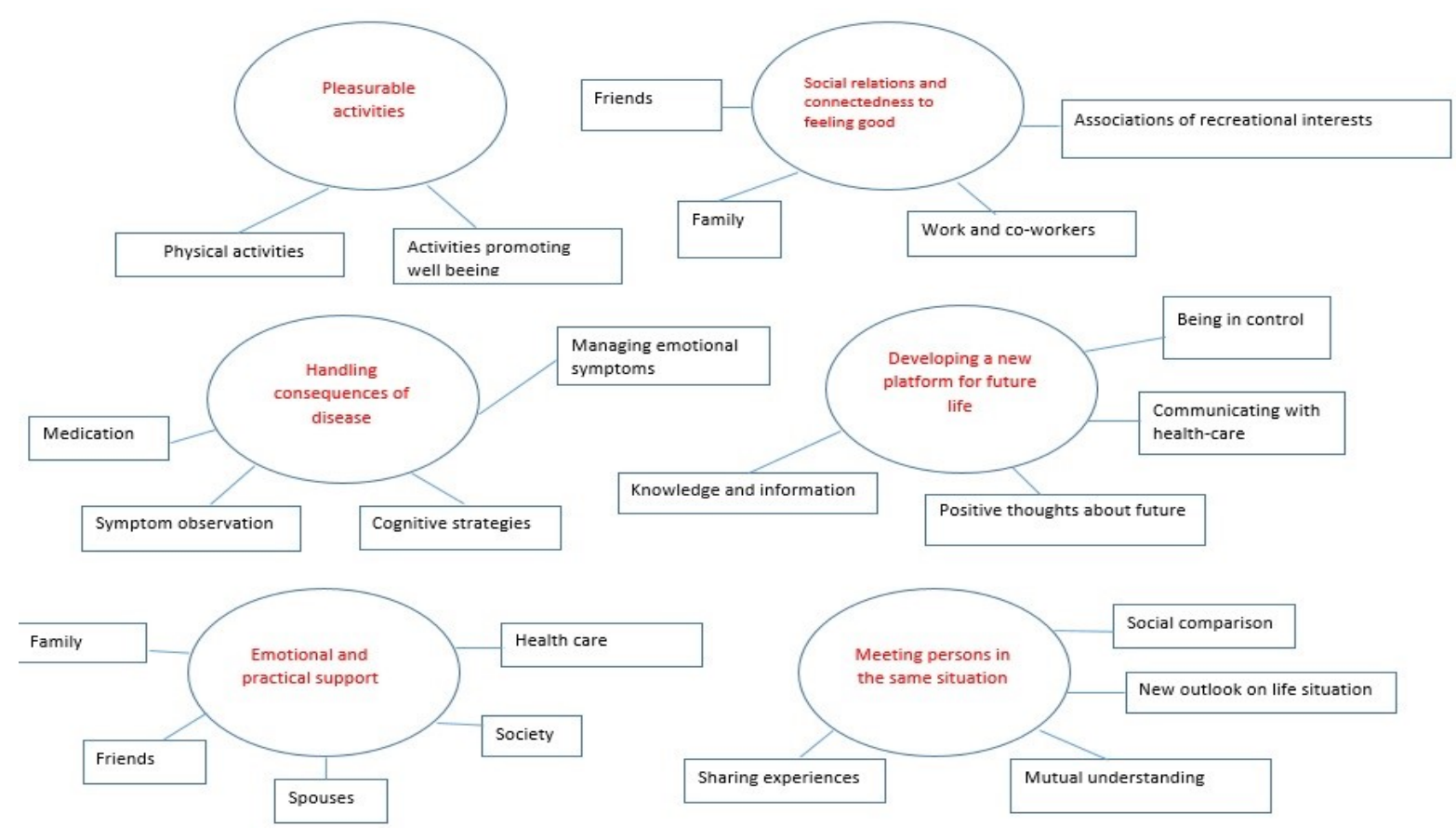

Figure 2: 


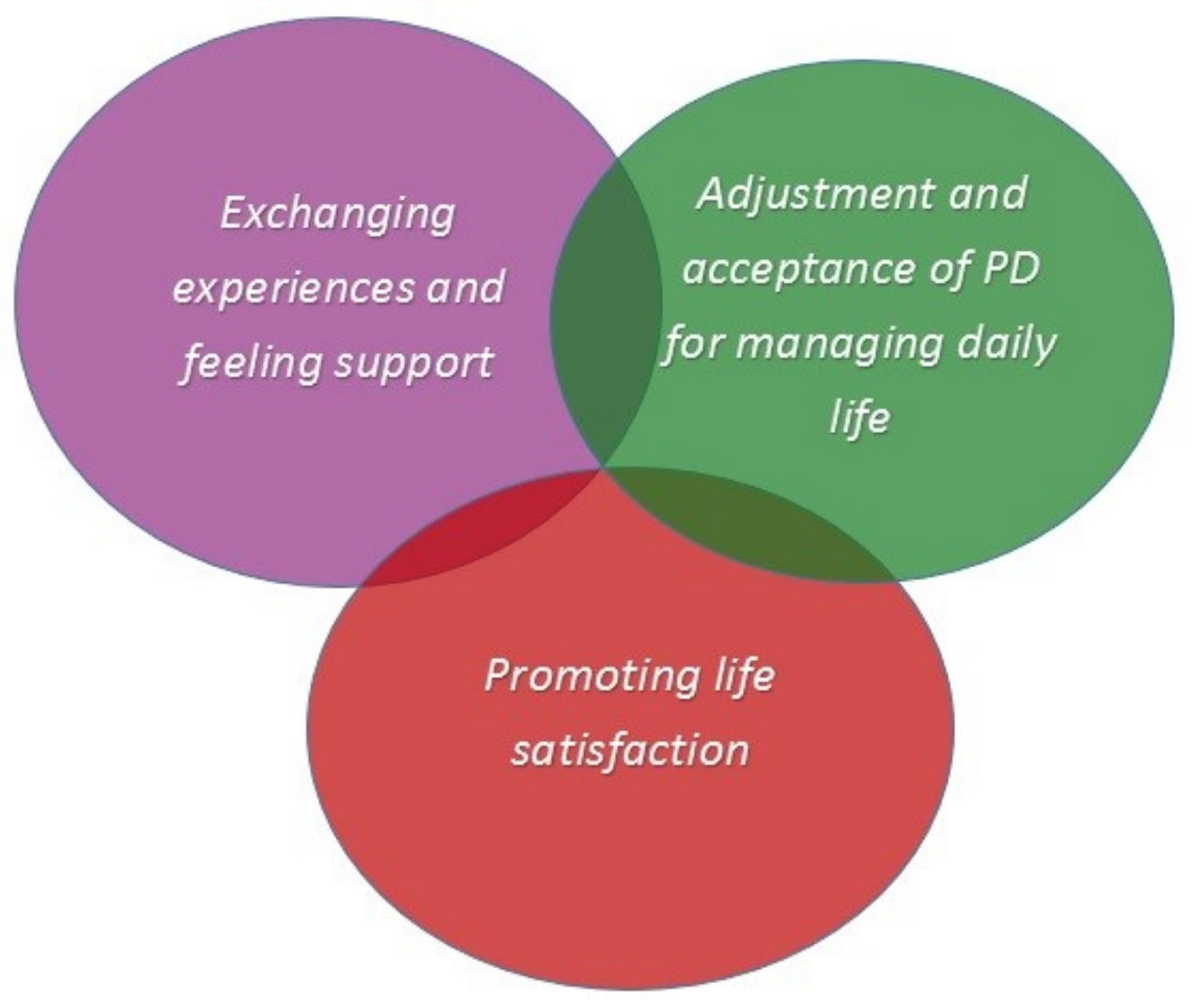

Figure 3: 


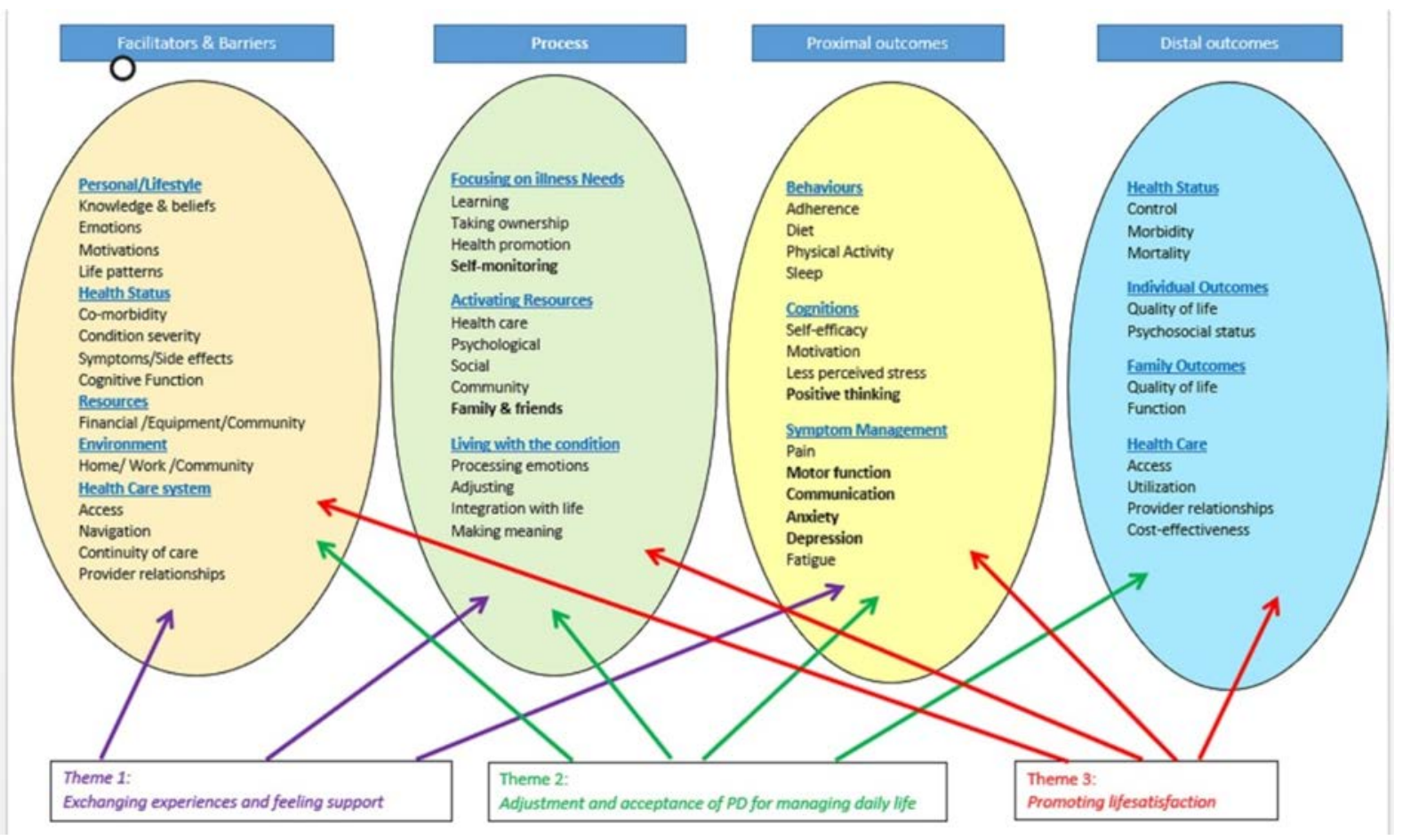



Nationella Parkinsonskolan (NPS), in English National Parkinson School, is a self-management program for people with Parkinson's and their families. NPS aims to provide people affected by Parkinson's and their families the knowledge and tools to enhance their ability to live and handle life with the chronic disease.

The program is based on the knowledge and experience gained from cognitive behavioral therapy. By promoting awareness of the possible thoughts, feelings and actions in relation to the
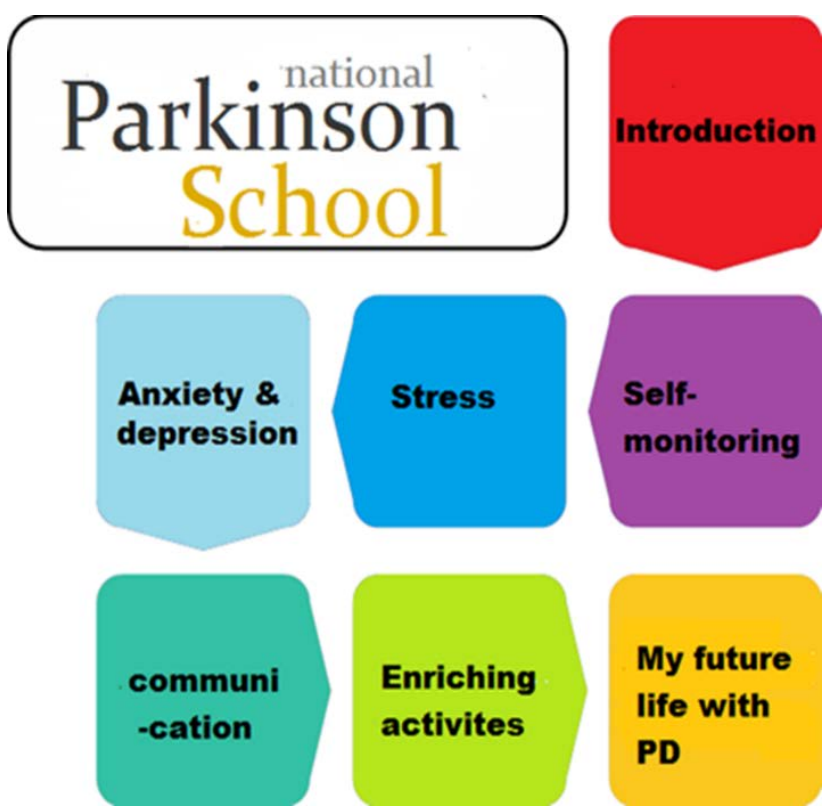

My future life with PD impact of disease in daily life and by introducing techniques of self-monitoring and self observations participants of the NPS get the tools needed to initiate life changes. Replacing negative thoughts with constructive thoughts helps manage difficulties and leads to a more positive perception of the living situation and provide a better quality of life.

The NPS does not focus on the disease itself, but on how to live a good life in spite of the presece of Parkinson's disease. The program is based on the idea that the participants first need knowledge about the disease itself and an understanding on how it can affect their lives. Knowledge and self-awareness is the platform to accept and understand the changing life situation. A new awareness about their own thoughts and reactions can help manage challenges in a better way. How people with PD and their families choose to relate to the disease and the changing life situation greatly affects the ability to maintain a good quality of life, despite the difficulties.

The seven themes of the NPS are presented in the figure above 